\title{
Plasmon confinement in fractal quantum systems
}

\author{
Tom Westerhout, ${ }^{2}$ Edo van Veen, ${ }^{2,1}$ Mikhail I. Katsnelson, ${ }^{2}$ and Shengjun Yuan ${ }^{1,2, \text { * }}$ \\ ${ }^{1}$ School of Physics and Technology, Wuhan University, Wuhan 430072, China \\ ${ }^{2}$ Institute for Molecules and Materials, Radboud University, NL-6525 AJ Nijmegen, The Netherlands
}

(Dated: January 22, 2018)

\begin{abstract}
Recent progress in the fabrication of materials has made it possible to create arbitrary non-periodic two-dimensional structures in the quantum plasmon regime. This paves the way for exploring the plasmonic properties of electron gases in complex geometries such as fractals. In this work, we study the plasmonic properties of Sierpinski carpets and gaskets, two prototypical fractals with different ramification, by fully calculating their dielectric functions. We show that the Sierpinski carpet has a dispersion comparable to a square lattice, but the Sierpinski gasket features highly localized plasmon modes with a flat dispersion. This strong plasmon confinement in finitely ramified fractals can provide a novel setting for manipulating light at the quantum scale.
\end{abstract}

Nowadays, different experimental techniques allow for the creation of arbitrary non-periodic two-dimensional (2D) lattices. For example, artificial lattices [1, 2], systems consisting of quantum dots that can be arranged in any custom shape, have attracted a lot of attention lately. More generally, nanolithography methods can be used to make high-quality 2D structures of arbitrary shape with a resolution in the order of tens of nanometers [3]. Other methods, such as molecular self-assembly [4, 5] have been used to grow Sierpinski gaskets. This presents an opportunity to experimentally study complex $2 \mathrm{D}$ systems, such as fractals.

Fractals have no translational invariance, so where a Bloch description is natural in the case of lattices, here it is not possible. Still, the Schrödinger equation has been solved analytically on some simple fractals with finite ramification [6]. For others, like the Sierpinski carpet, no analytical expressions for eigenenergies and eigenstates have been found yet. The latter systems are better tackled numerically [7]. It has been shown that the quantum conductance of Sierpinski carpets exhibits fractal fluctuations [8] and that their optical conductivity features sharp peaks due to electronic state pairs at characteristic length scales present within the carpet [9]. However, its plasmonic properties have not been investigated yet.

Historically, in most plasmonic devices, the Fermi wavelength of the electrons was much smaller than the plasmon wavelength which is of the order of the geometric size of the system for standing waves. In other words, the characteristic plasmon wave vector $q \ll k_{F}$, where $k_{F}$ is the Fermi wave vector. In this regime, plasmons can be described classically and there is no need to use a quantum mechanical approach [15 18].

Recently, due to the progress in nanodevice fabrication, the quantum regime for plasmons has been reached [19, 20]. In this regime, localized surface plasmons make it possible to confine light to scales much smaller than the scales of conventional optics, and as such provide a unique way for light manipulation on scales below the diffraction limit. Surface plasmons have found applications in surface-enhanced spectroscopy [10, 11], biological and chemical sensing [12], lithographic fabrication [13], and photonics [14].

However, the theory of inhomogeneous quantum electron plasma, even in the simplest random-phase approximation (RPA) [15 18], is quite complicated due to the essential nonlocality of the dielectric function 17]. Recently, a rigorous scattering theory of plasmons by obstacles was built 21], but finding plasmon eigenmodes of inhomogeneous quantum systems still remains a challenge. As a matter of fact, this problem is very old, starting with the early considerations [22, 23] of "atomic plasmons" 24 28] which eventually turned out to not exist [29, 30]. Previous attempts use additional uncontrollable approximations such as truncation of quantum states [28], semi-classical [24, 27, 30] or even classical [26] approaches.

Here we will present the results of accurate, straightforward calculations of plasmon spectra in an inhomogeneous quantum system with nontrivial geometry, namely Sierpinski carpets and gaskets, two prototypic examples of infinitely and finitely ramified fractals, respectively. These two types of fractals can have widely different properties. For example, it has been found that infinitely ramified fractals exhibit phase transitions not present in finitely ramified fractals [31].

In this letter first we outline the methods used and present a numerical method for calculation of plasmonic properties of systems with no translational invariance that is applicable to arbitrary geometries. Then, we discuss the results of these calculations on fractal systems. We compare the plasmon dispersions of the Sierpinski carpet and gasket to those of a square and triangle, respectively.

We consider a system described by a tight-binding Hamiltonian

$$
\hat{H}=-t \sum_{\langle a, b\rangle} \hat{c}_{a}^{\dagger} \hat{c}_{b}
$$

where $t$ is the hopping parameter. Here, we have taken the on-site potential to be zero and only consider nearest- 
neighbor hoppings. The two systems of interest are illustrated in Fig. [1]

(a)

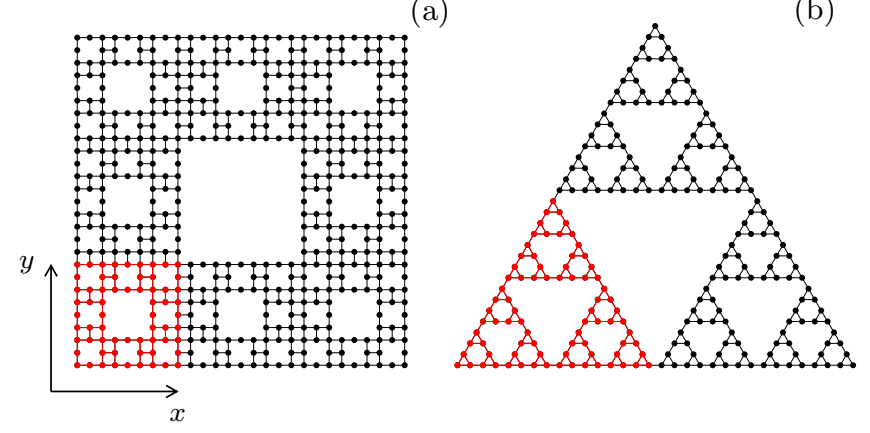

FIG. 1. The fractals considered in this paper. (a) A third iteration Sierpinski carpet. The width of the sample is $3^{3}=27$ lattice constants, or approximately $6.6 \mathrm{~nm}$. (b) A fifth iteration Sierpinski gasket. Its width is $2^{5}=32$ lattice constants $(7.9 \mathrm{~nm})$. The previous iterations are indicated in red.

Fractals are made using an iterative process. For example, to make the Sierpinski carpet, a previous iteration (indicated in red in Fig. 1) is copied $\mathcal{N}=8$ times to make a next iteration that is $\mathcal{L}=3$ times wider. With each fractal we can associate a Hausdorff dimension, given by $d_{H}=\log _{\mathcal{L}} \mathcal{N}$, as a measure for how space-filling its structure is. For the carpet $d_{H} \approx 1.89$, for the gasket $d_{H} \approx 1.58$.

Moreover, for each fractal we can define a ramification number, giving a measure of how connected it is. The Sierpinski carpet is infinitely ramified: as a higher iteration is taken, the number of bonds that need to be cut to separate it from a lower iteration goes to infinity. In contrast, the Sierpinski gasket is finitely ramified.

We use a hopping parameter $t=2.8 \mathrm{eV}$ and a lattice constant $a=0.246 \mathrm{~nm}$. These are the parameters for graphene, and they are representative for 2D systems in general. Choosing a different lattice constant will lead to a different plasmon spectrum, but the same qualitative behaviour.

Using this tight-binding model we obtain the exact eigenstates $|i\rangle$ with corresponding eigenenergies $E_{i}$, to use for the calculation of the dielectric function.

The dielectric function operator $\hat{\varepsilon}(\omega)$, by definition, relates the external potential $\hat{V}_{\text {ext }}(\omega)$ to the total potential $\hat{V}$ :

$$
\left\langle\mathbf{r}\left|\hat{V}_{\text {ext }}(\omega)\right| \mathbf{r}\right\rangle=\int \mathrm{d}^{d} r^{\prime}\left\langle\mathbf{r}|\hat{\varepsilon}(\omega)| \mathbf{r}^{\prime}\right\rangle\left\langle\mathbf{r}^{\prime}|\hat{V}| \mathbf{r}^{\prime}\right\rangle
$$

$d$ is the dimension of our problem. For the systems considered here $d=2$. Treating $\hat{V}$ as a perturbation, within RPA, the dielectric function may be expressed as follows
[17]:

$$
\begin{aligned}
\left\langle\mathbf{r}|\hat{\varepsilon}(\omega)| \mathbf{r}^{\prime}\right\rangle & =\left\langle\mathbf{r} \mid \mathbf{r}^{\prime}\right\rangle-\int \mathrm{d}^{d} r^{\prime \prime}\left\langle\mathbf{r}\left|\hat{V}_{\mathrm{C}}\right| \mathbf{r}^{\prime \prime}\right\rangle\left\langle\mathbf{r}^{\prime \prime}|\hat{\chi}(\omega)| \mathbf{r}^{\prime}\right\rangle, \\
\left\langle\mathbf{r}\left|\hat{V}_{\mathrm{C}}\right| \mathbf{r}^{\prime \prime}\right\rangle & \equiv \frac{e^{2}}{\left\|\mathbf{r}-\mathbf{r}^{\prime \prime}\right\|}, \\
\left\langle\mathbf{r}^{\prime \prime}|\hat{\chi}(\omega)| \mathbf{r}^{\prime}\right\rangle & =g_{s} \cdot \lim _{\eta \rightarrow 0+} \sum_{i, j}\langle i|\hat{G}| j\rangle\left\langle j \mid \mathbf{r}^{\prime \prime}\right\rangle\left\langle\mathbf{r}^{\prime \prime} \mid i\right\rangle\left\langle i \mid \mathbf{r}^{\prime}\right\rangle\left\langle\mathbf{r}^{\prime} \mid j\right\rangle, \\
\langle i|\hat{G}| j\rangle & \equiv \frac{n_{i}-n_{j}}{E_{i}-E_{j}-\hbar(\omega+i \eta)} .
\end{aligned}
$$

$|\mathbf{r}\rangle$ denotes a position eigenvector; $\hat{V}_{\mathrm{C}}$ is the Coulomb interaction potential; $\hat{\chi}(\omega)$ is the polarizability function; $\eta$ is the inverse relaxation time; $g_{s}=2$ is spin degeneracy; $n_{i}$ is $i$ 'th energy level occupational number according to the Fermi-Dirac distribution

$$
n_{i}=\frac{1}{e^{\left(E_{i}-\mu\right) / k T}+1} .
$$

We used room temperature $T=300 \mathrm{~K}$ and an inverse relaxation time $\eta=6 \mathrm{meV} / \hbar$.

Eqs. (3) allow us to exactly calculate the full dielectric function $\hat{\varepsilon}(\omega)$ of any tight-binding system without translational invariance. The open source project documentation [33] lists the computational techniques employed which, despite the $\mathcal{O}\left(N^{4}\right)$ algorithmic complexity, make calculations possible for systems of up to several thousands of sites.

To visualise the plasmon modes in a quantum mechanical system Wang et al [34] introduced the following method. Consider the dielectric function in its spectral decomposition:

$$
\hat{\varepsilon}(\omega)=\sum_{n} \epsilon_{n}(\omega)\left|\phi_{n}(\omega)\right\rangle .
$$

In this method, for each $\omega$ we consider only the eigenvalue $\epsilon_{n_{1}(\omega)}(\omega)$ that has the highest value of $-\operatorname{Im}\left[1 / \epsilon_{n}(\omega)\right]$, which gives us the plasmon eigenmode $\left|\phi_{n_{1}(\omega)}(\omega)\right\rangle$ that contributes most to the loss function.

However, it is not clear how to access these plasmon modes experimentally. Currently, the standard way of probing plasmon properties of small quantum mechanical systems is EELS. The fact that we calculate the full dielectric function gives us the possibility to calculate the following Fourier transform, which distinguishes this study from others:

$$
\langle\mathbf{q}|\hat{\varepsilon}(\omega)| \mathbf{q}\rangle=\frac{1}{(2 \pi)^{d}} \int \mathrm{d}^{d} r \int \mathrm{d}^{d} r^{\prime}\left\langle\mathbf{r}|\hat{\varepsilon}(\omega)| \mathbf{r}^{\prime}\right\rangle e^{-i \mathbf{q}\left(\mathbf{r}-\mathbf{r}^{\prime}\right)} .
$$

The loss function $-\operatorname{Im}[1 /\langle\mathbf{q}|\hat{\varepsilon}(\omega)| \mathbf{q}\rangle]$ is then directly measurable using EELS techniques [15 18, 35].

Formally, there are two ways of identifying plasmons. A plasmon frequency is either given by a local maximum of the loss function $-\operatorname{Im}\left[1 / \epsilon_{n_{1}(\omega)}(\omega)\right]$, or by a frequency 

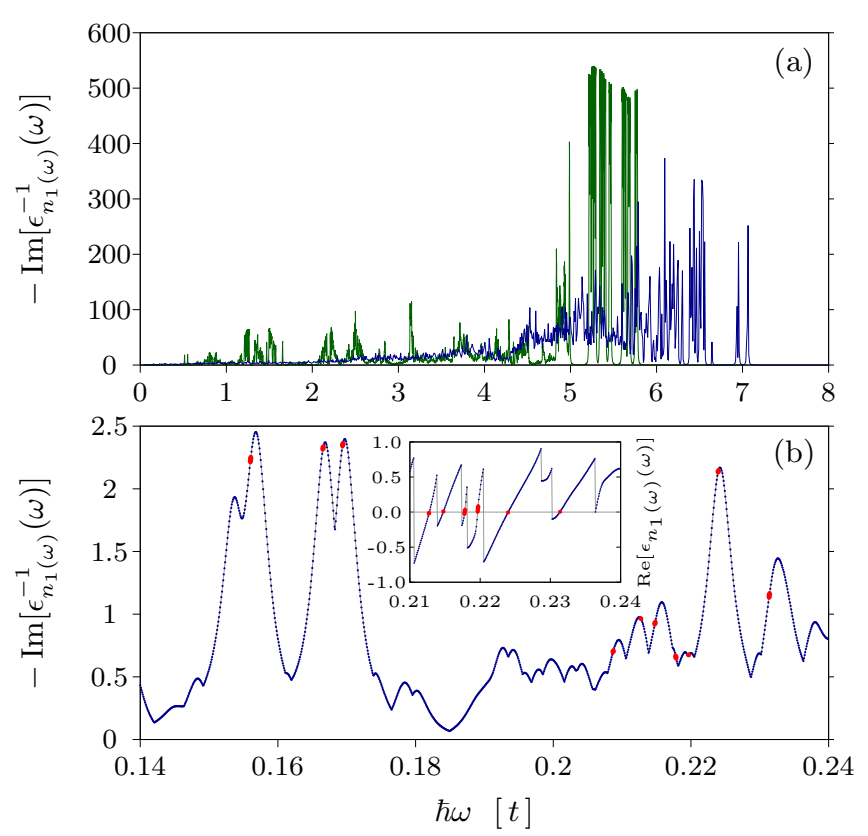

FIG. 2. The highest contribution to the loss function $-\operatorname{Im}\left[\epsilon_{n_{1}(\omega)}^{-1}(\omega)\right]$. (a) The loss function for the entire range of frequencies, in the case of (blue) a third iteration Sierpinski carpet and (green) a sixth iteration Sierpinski gasket. (b) The loss function of a third iteration Sierpinski carpet for a frequency interval $0.14 t<\hbar \omega<0.24 t$. Inset: $\operatorname{Re}\left[\epsilon_{n_{1}(\omega)}(\omega)\right]$ for a frequency interval $0.21 t<\hbar \omega<0.24 t$, showing discontinuities. Red dots indicate pairs of points between which $\operatorname{Re}\left[\epsilon_{n_{1}(\omega)}(\omega)\right]$ crosses zero in a continuous manner.

at which $\operatorname{Re}\left[\epsilon_{n_{1}(\omega)}(\omega)\right]=0$. These frequencies are not exactly equal due to Landau damping, which is quantified by $\eta$ [32].

The real-space loss function of the highest contributing plasmon mode is shown in Fig. 2, It shows that there is a large number of plasmon frequencies, and that the associated losses increase with increasing frequency. At each discontinuity in $\operatorname{Re}\left[\epsilon_{n_{1}(\omega)}(\omega)\right]$ a different mode is found to be the highest contributor to the loss function. Such a discontinuity is not associated with a plasmon, even though $\operatorname{Re}\left[\epsilon_{n_{1}(\omega)}(\omega)\right]$ switches sign.

The real part of the highest contributing plasmon eigenmodes for both the carpet and gasket are shown in Fig. 3, For further analysis, the inverse participation ratio $\operatorname{IPR}(\omega)=\int \mathrm{d}^{d} r\left|\left\langle\mathbf{r} \mid \phi_{n_{1}(\omega)}\right\rangle\right|^{4}$ can give us a measure of localization. The average IPR of $\left|\phi_{n_{1}(\omega)}\right\rangle$ was found to be an order of magnitude higher for the gasket than for the carpet. This can be seen as a consequence of the finite ramification of the gasket, i.e. the fact that it is less connected, and therefore the electrons are more confined and exhibit more localized plasmon eigenmodes. Fig. 3(d) shows an example of such a highly localized mode.

We now turn to the Fourier transform of the real-space loss function in order to make a comparison to EELS experiments. Fig. 4 shows the loss function as function

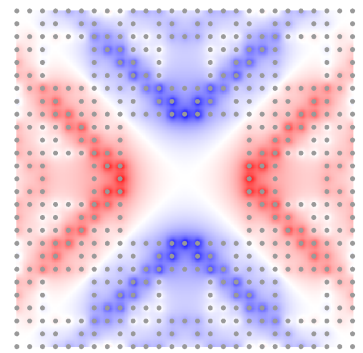

(a) $\hbar \omega=0.166518 t$

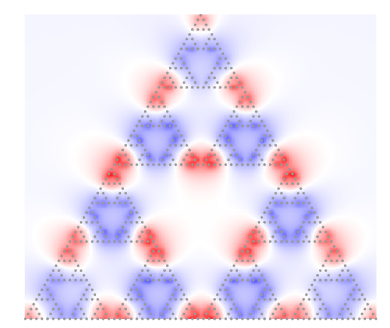

(c) $\hbar \omega=0.814286 t$

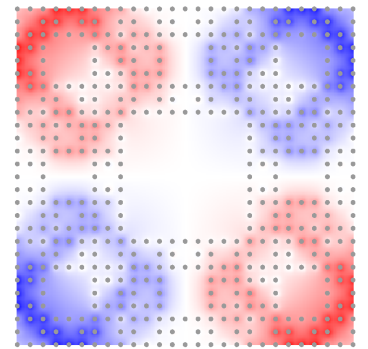

(b) $\hbar \omega=0.169375 t$

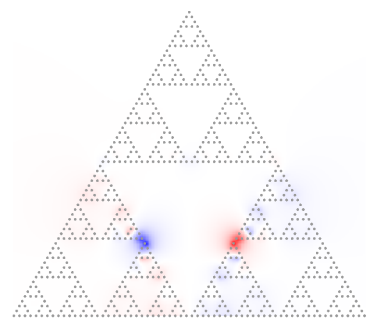

(d) $\hbar \omega=0.939286 t$
FIG. 3. The highest contributing plasmon eigenmodes in real space. A few examples of the real space distribution $\operatorname{Re}\left[\left\langle\mathbf{r} \mid \phi_{n_{1}(\omega)}(\omega)\right\rangle\right]$ of plasmon modes for $(\mathrm{a}, \mathrm{b})$ a third iteration Sierpinski carpet and (c,d) a sixth iteration Sierpinski gasket. Eigenmodes exhibiting different characteristic length scales are shown.

of both $q$ and $\omega$.

There is a close resemblance between the carpet (Fig. 4(a)) and a square sample (Fig. 4(b)). The dispersion of the carpet has extra broadening, similar to the broadening found in systems with disorder 36]. However, generally speaking, both curves look like a regular $\varepsilon(\omega) \propto \sqrt{q}$ dispersion relation for surface plasmons [18]. The carpet exhibits no translational invariance, i.e. $q$ is not actually a good quantum number, so this behavior is quite remarkable. The dispersion of the fourth iteration Sierpinski carpet is already very close to the third iteration dispersion. This convergence indicates that the result is representative for the real fractal at infinite iteration.

For the Sierpinski gasket (Fig. 廿4(c)), we observe different behavior. This fractal does not closely follow the dispersion relation of a triangle built out of a triangular lattice (Fig. 4(d)). Instead, we can clearly see the formation of multiple localized modes with near flat dispersion. Again, this result is reasonably converged.

Concluding, in this work we have calculated the plasmon dispersion for the Sierpinski carpet and Sierpinski gasket. The Sierpinski carpet has a plasmon dispersion comparable to the dispersion of a square lattice, whereas the gasket exhibits highly localized plasmon modes. More generally, a finitely ramified fractal can exhibit strong plasmon confinement, providing a novel setting for the manipulation of light at the quantum scale. With current experimental techniques, these results can be probed experimentally. Moreover, we have 


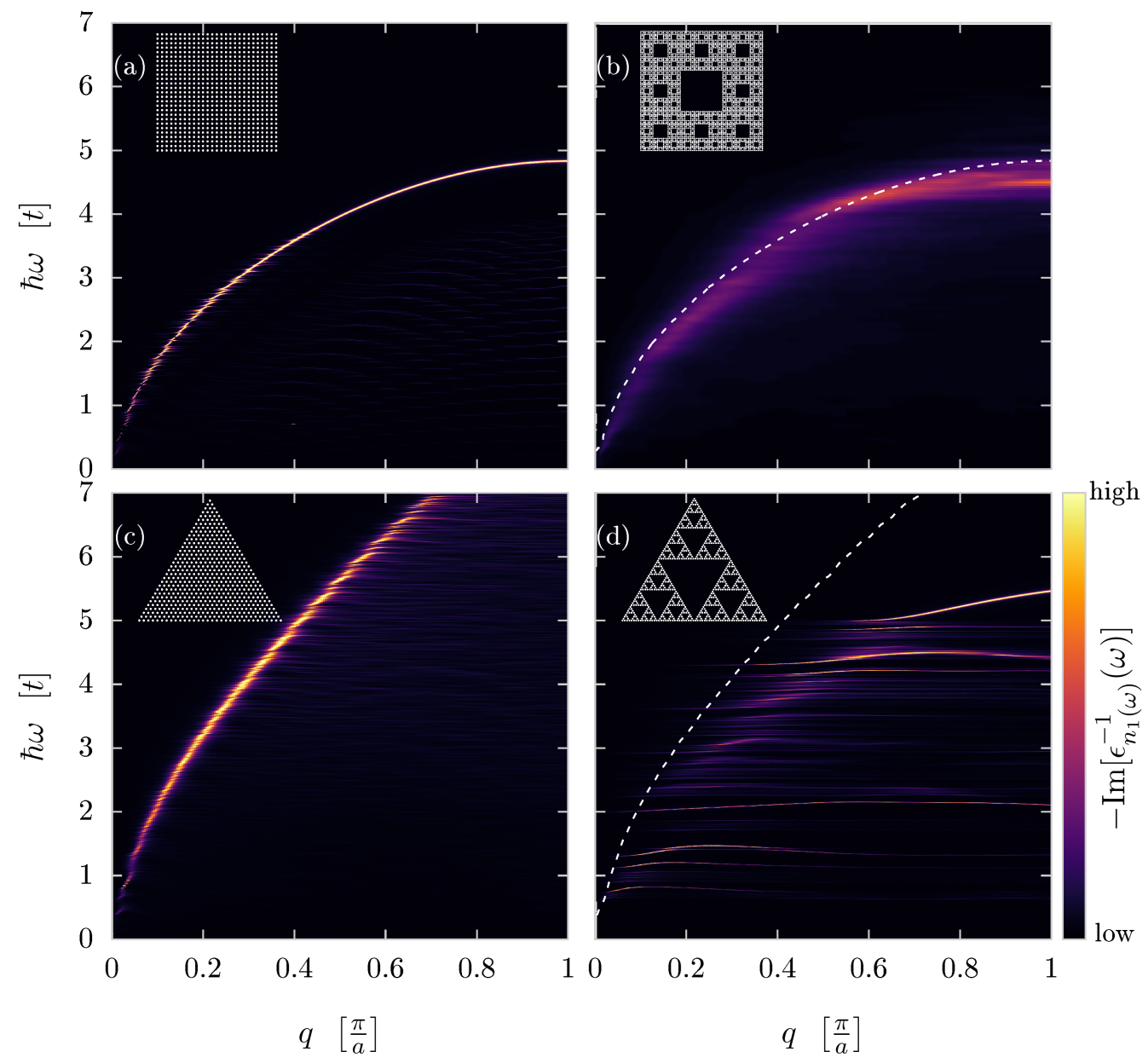

FIG. 4. Dispersion relation $-\operatorname{Im}[1 /\langle\mathbf{q}|\hat{\varepsilon}(\omega)| \mathbf{q}\rangle]$, showing the frequency and momentum dependency of the loss function. Momentum was taken along the $x$-axis. (a) A square built out of square lattice as compared to (b) the fourth iteration Sierpinski carpet. Similarly, (c) a triangle built out of triangular lattice as compared to (d) a sixth iteration Sierpinski gasket. The maximum of the left hand side is plotted as a dashed white line on the right hand side.

presented a rigorous approach for calculating plasmonic properties of generic tight-binding systems, published as an open source software project [33]. We believe that this code can be very useful for future projects relating to plasmonic properties of non-translationally invariant systems.

This work was supported by the National Science Foundation of China under Grant No. 11774269 and by the Dutch Science Foundation NWO/FOM under grant No. 16PR1024 (S.Y.), and by the European Research Council Advanced Grant program (contract 338957) (M.I.K.). Support by the Netherlands National Computing Facilities foundation (NCF), with funding from the Netherlands Organisation for Scientific Research (NWO), is gratefully acknowledged.

\footnotetext{
s.yuan@whu.edu.cn
}

[1] M. Gibertini, A. Singha, V. Pellegrini, M. Polini, G. Vignale, A. Pinczuk, L. N. Pfeiffer, and K. W. West, Phys. Rev. B 79, 241406 (2009).

[2] M. Polini, F. Guinea, M. Lewenstein, H. C. Manoharan, and V. Pellegrini, Nature nanotechnology 8, 625 (2013).

[3] D. Scarabelli, S. Wang, A. Pinczuk, S. J. Wind, Y. Y. Kuznetsova, L. N. Pfeiffer, K. West, G. C. Gardner, M. J. Manfra, and V. Pellegrini, Journal of Vacuum Science \& Technology B, Nanotechnology and Microelectronics: Materials, Processing, Measurement, and Phenomena 33, 06FG03 (2015).

[4] G. R. Newkome, P. Wang, C. N. Moorefield, T. J. Cho, P. P. Mohapatra, S. Li, S.-H. Hwang, O. Lukoyanova, L. Echegoyen, J. A. Palagallo, et al., Science 312, 1782 (2006).

[5] J. Shang, Y. Wang, M. Chen, J. Dai, X. Zhou, J. Kuttner, G. Hilt, X. Shao, J. M. Gottfried, and K. Wu, Nature Chemistry 7, 389 (2015).

[6] E. Domany, S. Alexander, D. Bensimon, and L. P. Kadanoff, Phys. Rev. B 28, 3110 (1983).

[7] M. Perreau, J. Peiro, and S. Berthier, Phys. Rev. E 54, 4590 (1996).

[8] E. van Veen, S. Yuan, M. I. Katsnelson, M. Polini, and 
A. Tomadin, Phys. Rev. B 93, 115428 (2016)

[9] E. van Veen, A. Tomadin, M. Polini, M. I. Katsnelson, and S. Yuan, Physical Review B 96, 235438 (2017).

[10] C. L. Haynes, C. R. Yonzon, X. Zhang, and R. P. Van Duyne, Journal of Raman Spectroscopy 36, 471 (2005)

[11] A. C. R. Pipino, G. C. Schatz, and R. P. Van Duyne, Phys. Rev. B 49, 8320 (1994).

[12] C. R. Yonzon, D. A. Stuart, X. Zhang, A. D. McFarland, C. L. Haynes, and R. P. V. Duyne, Talanta 67, 438 (2005), nanoscience and Nanotechnology.

[13] W. Srituravanich, N. Fang, C. Sun, Q. Luo, and X. Zhang, Nano letters 4, 1085 (2004).

[14] M. L. Brongersma and P. G. Kik, Surface Plasmon Nanophotonics (Springer, 2007).

[15] P. Nozières and D. Pines, Theory of Quantum Liquids (Hachette UK, 1999).

[16] P. M. Platzman and P. A. Wolff, Waves and Interactions in Solid State Plasmas (Academic Press, 1973).

[17] S. V. Vonsovsky and M. I. Katsnelson, Quantum Solidstate Physics (Springer Verlag, 1989).

[18] G. Giuliani and G. Vignale, Quantum Theory of the Electron Liquid, Masters Series in Physics and Astronomy (Cambridge University Press, 2005).

[19] J. A. Scholl, A. L. Koh, and J. A. Dionne, Nature 483, 421 (2012).

[20] M. S. Tame, K. McEnery, Ş. Özdemir, J. Lee, S. A. Maier, and M. S. Kim, Nature Physics 9, 329 (2013).

[21] I. Torre, M. I. Katsnelson, A. Diaspro, V. Pellegrini, and
M. Polini, Physical Review B 96, 035433 (2017).

[22] F. Bloch, Zeitschrift für Physik 81, 363 (1933).

[23] H. Jensen, Zeitschrift für Physik 106, 620 (1937).

[24] B. K. Ishmukhametov, Phys. stat sol.(b) 45, 669 (1971).

[25] A. Sen, Lettere Al Nuovo Cimento (1971-1985) 8, 749 (1973).

[26] G. V. Gadiyak, A. Kirzhnits, and E. Lozovik, Zh. Eksp. Teor. Fiz 69, 122 (1975).

[27] B. K. Ishmukhametov and M. I. Katsnelson, Fiz. Met. Metalloved. 40, 736 (1975).

[28] M. Y. Amusia and V. K. Ivanov, Physics Letters A 65, 217 (1978).

[29] E. T. Verkhovtseva, P. S. Pogrebnyak, and Y. M. Fogel, JETP Letters 24, 425 (1976).

[30] B. K. Ishmukhametov, M. I. Katsnelson, V. N. Larionov, and A. M. Ustjuzhanin, Physics Letters A 82, 387 (1981).

[31] Y. Gefen, A. Aharony, and B. B. Mandelbrot, Journal of Physics A: Mathematical and General 17, 1277 (1984).

[32] K. Andersen, K. W. Jacobsen, and K. S. Thygesen, Physical Review B 86, 245129 (2012).

[33] T. Westerhout, "Tools to calculate quantities related to plasmons in materials with no translational symmetry." https://github.com/twesterhout/plasmon-cpp (2017).

[34] W. Wang, T. Christensen, A.-P. Jauho, K. S. Thygesen, M. Wubs, and N. A. Mortensen, Scientific reports 5 (2015).

[35] J. Lu, K. P. Loh, H. Huang, W. Chen, and A. T. S. Wee, Physical Review B 80, 113410 (2009).

[36] F. Jin, R. Roldán, M. I. Katsnelson, and S. Yuan, Physical Review B 92, 115440 (2015). 